IBD

\title{
NEW PATHWAY IN IBD SUSCEPTIBILITY
}

New research from George Kollias and co-workers has implicated MAP3K8, via activation of the Cox-2-PGE 2 pathway, in intestinal homeostasis. This finding could have implications for the treatment of patients with IBD.

In 2012, a genome-wide association study revealed that a single nucleotide polymorphism in MAP $3 K 8$ was associated with IBD. "This finding was a strong indication for an unknown protective function for this gene in the intestine of humans," explains lead author Manolis Roulis. "We therefore decided to thoroughly investigate the mechanistic basis and the cellular specificities underlying the homeostatic function of MAP3K8 in the gut."

Mice with complete knockout of MAP3K8 were highly susceptible to colitis induced by dextran sodium sulphate. The knockout mice had increased weight loss, disease activity and lethality, as well as reduced colon length, compared with their wild-type littermates. Histopathological features of colitis were also more severe in the knockout mice than in the normal mice. Although the knockout mice had more tissue damage than the wild-type mice, they had similar levels of inflammation.

To examine the cell specificity of this response, the team generated mice in which MAP 3 K 8 was selectively inactivated in intestinal myofibroblasts. These mice had an exacerbated colitis phenotype, similar to that of the complete knockout mice, suggesting that inactivation of this gene in intestinal myofibroblasts is sufficient to increase susceptibility to colitis. These findings "identify a hitherto unsuspected homeostatic mechanism mediated by MAP3K 8 upon epithelial injury that is specific for intestinal myofibroblasts," concludes Roulis. Further experiments in mice demonstrated that intestinal myofibroblasts respond to epithelial injury by activating the Cox-2-PGE ${ }_{2}$ pathway via MAP3K8, which promotes intestinal homeostasis, and that this pathway is dysfunctional in IBD.

Roulis and co-workers also found that MAP3K8 expression was reduced in intestinal myofibroblasts from the inflamed ileum of nine patients with IBD. The authors suggest that their study "may also provide a basis for patient stratification and more precise future therapies."

Claire Greenhill

Original article Roulis, M. et al. Intestinal myofibroblastspecific Tpl2-Cox-2-PGE ${ }_{2}$ pathway links innate sensing to epithelial homeostasis. Proc. Natl Acad. Sci. USA doi:10.1073/pnas.1415762111 